Bull. Mater. Sci., Vol. 2, Number 2, May 1980, pp. 121-125. (C) Printed in India.

\title{
Dual anvil high pressure cell
}

\author{
A $K$ BANDYOPADHYAY, B S SIVARAM* and \\ S V SUBRAMANYAM \\ Department of Physics, Indian Institute of Science, Bangalore 560012 , India \\ * Present address : Department of Physics, University of North Eastern, Boston, \\ Massachusetts, USA.
}

MS received 16 January 1980

\begin{abstract}
A dual level high pressure cell using Bridgman anvils was constructed to facilitate simultaneous measurement on the samples. The anvils were of $4 \mathrm{~mm}$ and $5 \mathrm{~mm}$ diameter respectively. The anvils were made from tungsten carbide $3 \%$ cobalt binder). Pyrophyllite and steatite were used as the gasket and the pressure transmitting medium. The cell was calibrated against the polymorphic phase transition of bismuth and ytterbium.
\end{abstract}

Keywords. High pressure; dual level cell; calibration.

\section{Introduction}

In a previous paper (Bandyopadhyay et al 1980) we reported the clamp-type high pressure cell for carnying out electrical conductivity measurements on small solid samples at pressures upto $8 \mathrm{GPa}$ (i.e. 80 kilo bar) and for use down to $77 \mathrm{~K}$. In this paper, we report the dual level high pressure cell which can be used to generate similar pressures, i.e. upto $8 \mathrm{GPa}$, and in which two samples can be studied in the same run. This type of dual level cell was originally developed by (Fujii and Nagano 1975) and the present cell is a modified version of that cell.

\section{Experimental}

The cell consists of three Bridgman anvils shown in figure 1. These anvils were made from tungsten carbide (with 3\% cobalt binder). The first anvil has a tip diameter of $4 \mathrm{~mm}$, the middle one is $4 \mathrm{~mm}$ at the top and $5 \mathrm{~mm}$ at the bottom and the last one is $5 \mathrm{~mm}$. So, essentially, there are two different levels $4 \phi$ and $5 \phi$. This can be changed by suitably changing the anvil face. All these tips have a tapering angle of $12^{\circ}$ and are machined to a flatness better than $15 \mu$. The 

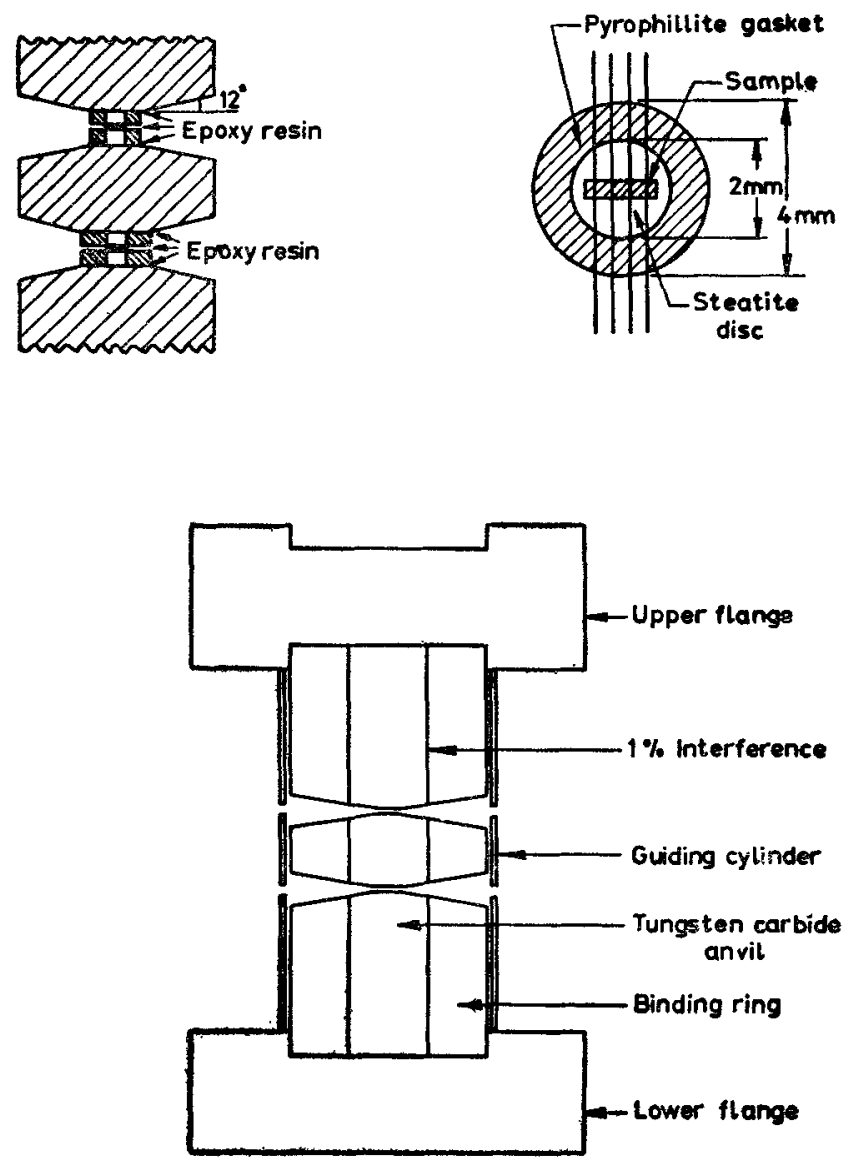

Figure 1. Schematic drawing of dual level cell and sample assembly.

anvils were strengthened by heat-treated EN 24 steel binding rings with 1\% interference and molybdenum disulphide was used as lubricant for insertion. A guiding cylinder enclosing the anvils and the binding rings helps their alignment. The guiding cylinder has diametrically opposite slots to facilitate viewing and for taking out electrical leads. The anvils were placed on a pair of flanges shown in the figure. The pressure is generated by a hydraulic press of $\mathrm{i} 0.0$ ton capacity and of ram diameter of $15 \mathrm{~cm}$. A Heise gauge $(0-1000$ psi) with a resolution of 1 psi was used to measure oil pressure. The sample assembly is shown in figure 1. Pyrophyllite was used as the gasket material with steatite as a pressure transmitting medium. The gasket rings of pyrophyllite (O.D. for the first and second levels was $4 \mathrm{~mm}$ and $5 \mathrm{~mm}$ respeztively, $I . D$. and thickness for both levels were $2 \mathrm{~mm}$ and $0.15 \mathrm{~mm}$ respectively) was heat-treated to increase the hardness. It was placed on one of the anvils with a mixture of araldite epoxy resin and ferric oxide facilitating an increased coefficient of friction between the two. The steatite disk (diameter $2 \mathrm{~mm}$ and thickness $0.15 \mathrm{~mm}$ ) which was made by 
pressing it in a die was placed in the central hole of the gasket ring and the specimen was mounted on it. A mixture of ferric oxide and resin was again used between the two gaskets and the whole assembly allowed to dry.

\section{Pressure calibration}

The pressures generated at room temperature were calibrated against the wellknown phase transition of bismuth (I-II) at $2.55 \mathrm{GPa}$, bismuth (II-III) at 2.69 GPa, ytterbium (fcc-bcc) at $4 \mathrm{GPa}$ and bismuth (IIL-V) at $7.7 \pm 0.3 \mathrm{GPa}$ (Lloyed 1971; Mcwhan et al 1969). The conventional four-probe method was used for electrical resistivity measurement with a Keithley constant current source (model No. 225) and DC null detector (model No. 155) as measuring instruments. Figure 2 shows the load versus normalised resistivity curve of bismuth and figure 3 is that of ytterbium. The resistances are expressed in terms of the resistance at normal atmospheric pressure. Figure 4 indicates the load versus generated pressure which reveals that the variation is almost linear. The transitions are quite sharp so that the homogeneity of the medium is quite satisfactory. Thus the insertion of one level does not affect as such but helps to reduce the experimental run by just one-half. The tip diameters of the first and second anvils are kept intentionally different $4 \mathrm{~mm}$ and $5 \mathrm{~mm}$ so that the pressure on the sample in the second anvil follows that in the first with a definite pressure lag. This enables careful and steady measurements near any phase transitions. This instrument has been used successfully to study the different organic charge transfer complexes which will be published elsewhere.

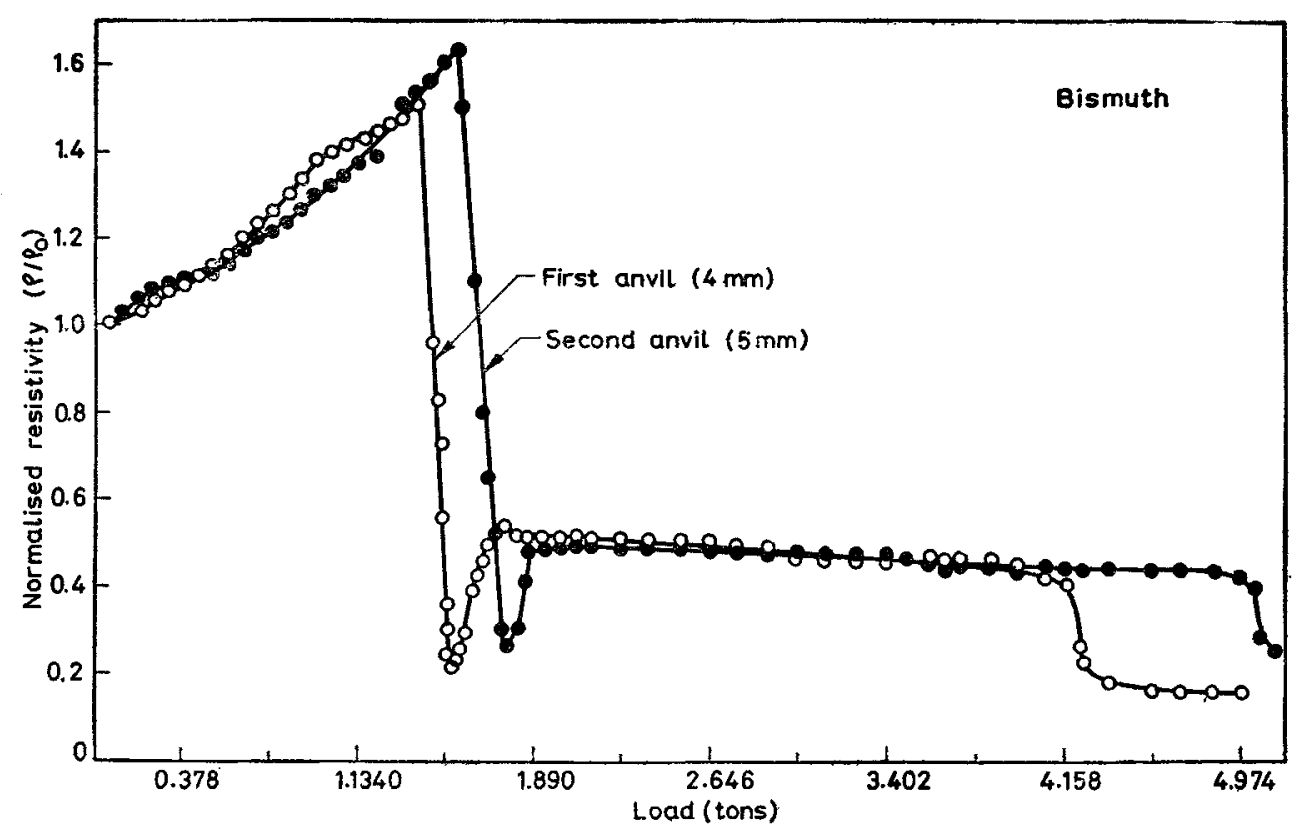

Figure 2. Normalised resistivity load curve for bismuth. 


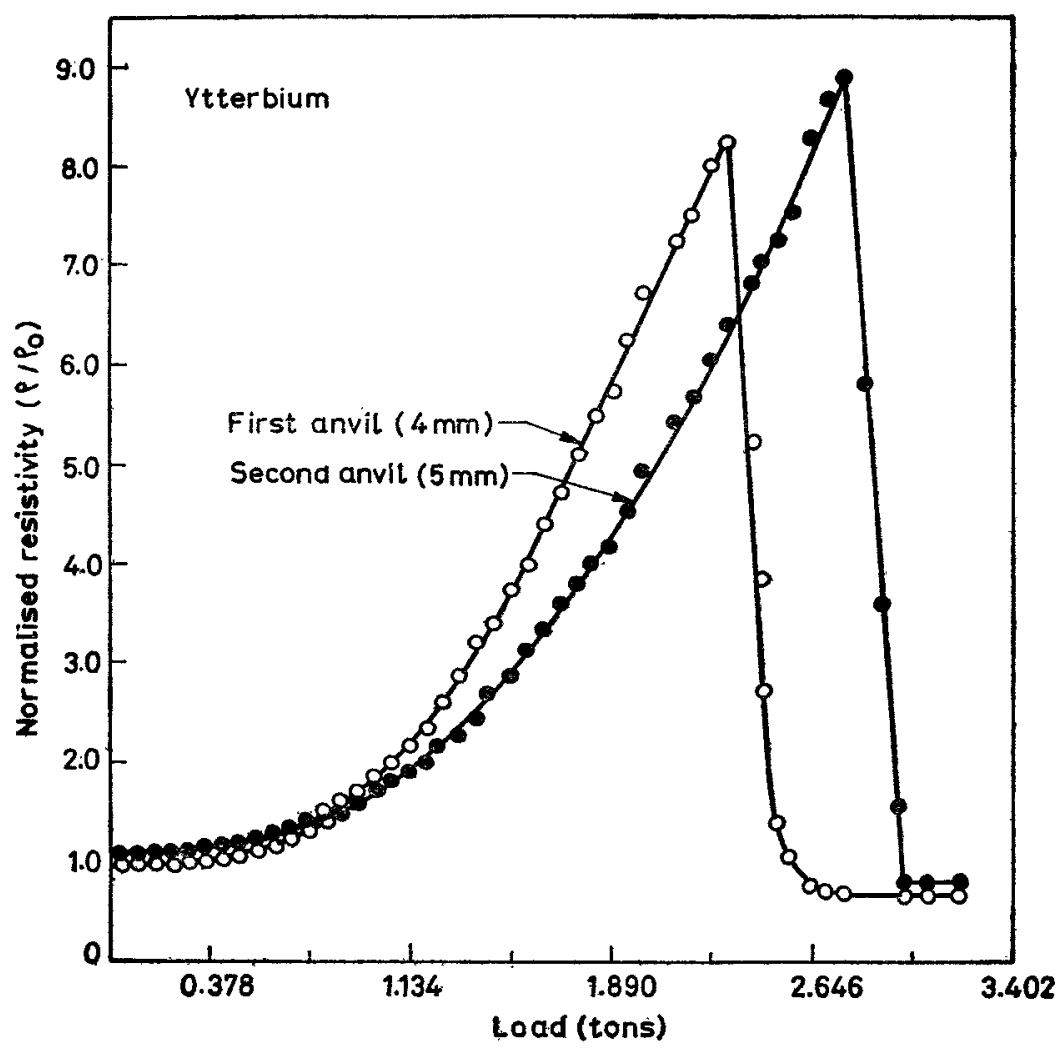

Figure 3. Normalised resistivity load curve for ytterbium.

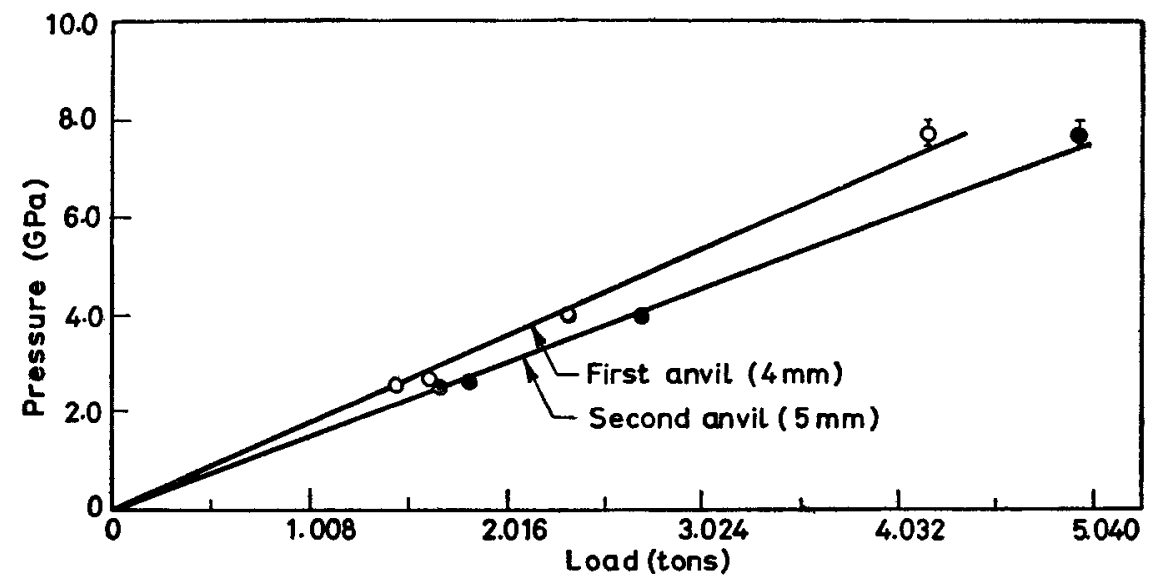

Figure 4. Pressure load curve. 


\section{Acknowledgements}

The authors are thankful to Dr A K Singh for his suggestions. They would like to asknowledge PL-480 and the Department of Ssience and Technology schemes for financial support.

\section{References}

Bandyopadhyay A K, Nalini A V, Gopal E S R and Subramanyam S V 1980 Rev. Sci. Instrum. 51136

Fujii G and Nagano H 1975 Jpn. J. Appl. Phys. 14919

Lloyed E C (ed.) 1971 NBS Symposium on accurate characterisation of high pressure environment, Pub. No. 326

Mcwhan D B, Rice T M and Schmidt P H 1969 Phys. Rev. 1771063. 